\title{
Nutrición Basada en Evidencia para la práctica comunitaria: entre la búsqueda de la cientificidad y la homogeneidad clínica.
}

\author{
Evidence-Based Nutrition for community practice: between searching scientificity and clinical \\ homogeneity.
}

Andrés Méndez Palacios Macedo ${ }^{a}$, Bernardo Adrián Robles Aguirre ${ }^{b}$

\begin{abstract}
:
For more than 30 years community practice in nutrition has pursued scientificity through the appliance of systematized search for information that can guide interventions appropriately. Notwithstanding the efforts, few are the results that have had a true impact on changing practices or had reflected a modification of the health and disease patterns of the population. In this work, different disciplines based on evidence are explored and the main theoretical concepts under which knowledge construction techniques have been developed, are questioned.
\end{abstract}

Keywords:

Evidence based nutrition, evidence based medicine, community nutrition, intercultural intervention, social medicine.

\section{Resumen:}

Durante más de 30 años se ha buscado dotar de cientificidad a la práctica comunitaria en nutrición a partir de implementar la sistematización de la información que pueda orientar las intervenciones de forma adecuada. No obstantes los esfuerzos, pocos son los resultados que hayan tenido un verdadero impacto en el cambio de las prácticas o reflejen una modificación de los perfiles de salud y enfermedad de la población. En este texto se exploran las diferentes disciplinas basadas en evidencia y se cuestionan los principales criterios bajo los cuales han sido desarrolladas las técnicas de construcción del conocimiento.

\section{Palabras Clave:}

Nutrición basada en evidencia, medicina basada en evidencia, nutrición comunitaria, intervención intercultural, medicina social.

\section{Introducción}

El panorama de salud y nutrición en México demuestra que, por más de una década, los retos que suponía la transición alimentaria no han podido ser superados: las prevalencias de sobrepeso y obesidad, junto con la coexistencia de enfermedades carenciales y metabólicas implican que las demandas específicas para los nutriólogos requieren abarcar un amplio espectro de conocimientos y habilidades que, lejos de ser aplicados de forma integral, deben están sujetos a una revisión constante.

La Medicina Basada en Evidencia (MBE) surgió a mediados de los años noventa (Sackett et al, 1996) como una herramienta que, desde un enfoque poblacional, pudiera orientar la toma de decisiones informadas en el ejercicio clínico de la profesión con el fin de mejorar los resultados sanitarios (Sacristán, 2013:460). Años más tarde, la Nutrición Basada en Evidencia (NuBE) se sumó a los esfuerzos por diversificar la MBE en otras áreas de especialidad. No obstante, a más de tres décadas de haberse desarrollado, los problemas en salud y nutrición prevalecen y se han vuelto cada vez más complejos.

Diversos han sido los señalamientos que desde las áreas académicas y empíricas han apuntado sobre los problemas de aplicación de la NuBE: conflicto de interés, verticalidad en el ejercicio de la intervención, mecanicismo y biologicismo. A propósito de las críticas, en los últimos diez años se han desarrollado otros esfuerzos por buscar mejores asideros para dar contenido a las buenas prácticas en donde se enfaticen los determinantes 
sociales de la salud (DDS)", la interacción con el sistema de salud de cada población y los elementos de interculturalidad presentes en la relación investigador, practicante y sujeto.

La práctica en Nutrición Comunitaria (NC) supone varios retos a la NuBE, puesto que las intervenciones, a diferencia de otras áreas de la salud y la nutrición, deberán contemplar el ejercicio profesional en un ámbito situado y ser sensible a las diferencias sociales, económicas, políticas y culturales de los sujetos. La falta en el desarrollo epistemológico de la profesión y el avance de los programas de estudios que sustraen la NC de sus currículos, representa un importante impedimento para promover una revisión crítica de la NuBE y una posterior reconfiguración, basada en la Práctica Basada en Evidencia (PBE), la Investigación Traslacional (IT) o las Ciencias de la Implementación $(\mathrm{Cl})$.

A continuación, se presenta un ejercicio de revisión de las necesidades del área profesional, buscando los principales conflictos en relación con la NuBE y las posibles soluciones basadas en otros métodos para buenas prácticas en la NC.

La argumentación se desarrolla desde los principios teóricos de la Medicina Social (López y Blanco, 1994), la Antropología Cultural de la Alimentación (Garine, 1999) y la Antropología Médica Crítica (Freyermuth y Sesia, 2006), bajo los cuales se busca evidenciar los principales supuestos: a) la nutrición es un componente del proceso salud-enfermedad-atención (PSEA), inserto en el modo de producción capitalista y que opera bajo la lógica neoliberal; desde esta perspectiva se asume como dinámico y determinado social e históricamente; b) la alimentación es un proceso social complejo que comprende a la nutrición y que está determinada tanto por componentes socioculturales como biológicos, siendo entonces sus expresiones bioculturales; c) la atención comprende sistemas de curación, restauración y manutención de la salud en contextos sociales, políticos y económicos; d) la NC implica contextos de interrelación entre personas y grupos con particularidades culturales; desde esta interacción, se posibilitan o limitan las posibilidades de resolución de los problemas de nutrición y alimentación.

El presente texto se divide en dos apartados: en el primero se revisan los antecedentes para definir la NuBE y se reconoce la necesidad de reconceptualizarla. En el segundo, se discute sobre superación de las bases teóricas de la epidemiología clínica para dar cuenta de los problemas relacionados con la nutrición comunitaria, cuyos derroteros escapan del espacio de las evidencias y

\footnotetext{
* Los DSS comprenden los comportamientos, estilos de vida, los ingresos, la posición social, la educación, el trabajo, las condiciones laborales, el acceso a servicios sanitarios adecuados y los entornos sobre la salud, todos ellos son importantes per se, están
}

la clínica. Hacia el final, dado que la tarea supera las posibilidades de la conclusión, se ofrecen algunas notas finales respecto a las prospectivas de las buenas prácticas en NC.

\section{Sobre la Nutrición Basada en Evidencia: breve reseña histórica y principales conceptos}

Foucault ha señalado al siglo XVIII como el espacio temporal para lo que denomina el nacimiento de la clínica, cuando la medicina convirtió a la salud en un objeto de intervención, dejando de lado a la enfermedad. La apropiación continuó hacia el cuerpo y contexto de las personas, haciendo del médico agente de control sobre los procesos cotidianos de su usuario, avalado por una comunidad y un Estado (Foucault, 1974). Este entramado, validado y reproducido es a lo que Foucault conoce en parte como biopolítica y se ha consolidado a propósito de esto, como una medicina hegemónica (Menéndez, 2003) En más de dos siglos, la medicalización, o la conversión en enfermedad de toda una serie de episodios vitales (Maldonado-Simó, 2012) ha avanzado de forma acelerada sobre el control del cuerpo y las prácticas humanas, involucrando aspectos antes desconocidos por las ciencias de la salud, como la sexualidad y la alimentación (Foucault, 1974; Masana, 2012).

El antecedente inmediato directo de la NuBE es la MBE, cuya práctica comenzó a despegar en 1996 desde la Universidad de McMaster en Canadá. La MBE/NuBE fue una respuesta a la tradición de la clínica, desde la cual los diagnósticos y tratamientos en salud, suponían la intervención vertical del médico sobre el cuerpo de las personas (Maestre, Ocampo, Useche y Trout, 2012). Desde esta perspectiva, la MBE/NuBE se erige como el cénit de las prácticas, puesto que imbrica los discursos científicos y las prácticas clínicas para un supuesto beneficio.

Uno de los preceptos fundamentales en la $\mathrm{MBE} / \mathrm{NuBE}$ es la beneficencia basada en las evidencias. Por beneficencia, se comprenden una gran diversidad de elementos de contenido ético-moral que se centran en el precepto aristotélico de "hacer bien". Sin embargo, a lo largo de la práctica se han hecho críticas particulares sobre el ejercicio médico centrado en la beneficencia, puesto que se asume como global, ahistórico, y apolítico (Alcántara-Moreno, 2008).

Por otra parte, el concepto central de la MBE/NuBE es la evidencia de la cual se busca dar contenido a la práctica profesional. Más adelante se

íntimamente relacionados entre sí, son acumulativos, causales y actúan en diferentes niveles: proximales, intermedios y estructurales (Liscano Pinzón y Montoya, 2015: 121). 
discutirán los aspectos relacionados con la conceptualización que sobre las evidencias ha realizado la medicina hegemónica.

A medida en que las críticas se hicieron presentes y a pesar de los esfuerzos por redirigir la MBE, se han propuesto otros modelos para dirigir la toma de decisiones clínicas. Algunos de estos trabajos han buscado mantener la esencia de la búsqueda por evidencias orientadoras de decisión mientras que otros, han luchado por superar los preceptos de la medicina hegemónica mediante la transdisciplina e interdisciplina (López y Blanco, 1994).

Aunque no siempre se han conducido de esta manera, para este trabajo se seguirán los conceptos esfuerzos del consenso* y esfuerzos críticos de López y Blanco (1994). En el primero se resalta la incorporación de las revisiones sistemáticas y los meta-análisis. La MBE/NuBE desde su desarrollo, se planteó la necesidad de procesar la información científica respecto a su relevancia, para lo cual ha propuesto diversos mecanismos de búsqueda y la validación científica externa. El objetivo principal fue identificar la calidad de la información, basándose en la identificación de sesgos y de tal forma, poder lograr recomendaciones objetivas.

Estos esfuerzos han conducido a la generación de métodos de búsqueda validados y de redes de expertos organizadas en plataformas electrónicas desde las cuales, se pueden simplificar las búsquedas de información y sus consecutivas, revisiones sistemáticas. Cabe señalar que uno de los motivos principales para realizar estos esfuerzos fue bajo la premisa de que el financiamiento de la investigación, en la mayoría de los casos, provenía de compañías farmacéuticas e intereses particulares gubernamentales (Del Olmo, Alcázar y López de Val, 2005).

Ya que no es de particular interés de este trabajo dar cuenta de cada uno de estos métodos, se recomienda revisar el Grading of Recommendations, Assesment, Development and Evaluation, CONSORT, TREND y PRISMA (Cobos-Aguilar, 2016; Maestre, Ocampo, Useche y Trout, 2012) y la plataforma de la Red Cochrane y el grupo JAMA (Gómez-Ortega y Amaya-Rey, 2013; Alexander, Andre y Fortes, 2018).

En este mismo grupo, se pueden encontrar a las redes de trabajo desarrolladas desde la perspectiva de las PBE, en las cuales comienza a incorporarse una perspectiva interdisciplinaria, pero alineada a los preceptos de la medicina hegemónica. Estos esfuerzos han incluido las revisiones sistemáticas como base de su trabajo, no obstante, han observado la importancia de recuperar la información de las prácticas profesionales en el campo de la salud. En el ámbito de la nutrición, resalta la Red NuBE en España, que es un sistema de recopilación de evidencias. Este grupo de trabajo se ha consolidado de tal forma que recientemente desarrollaron el software Practice-based Evidence in Nutrition (PEN), cuyo acceso con costo, permite realizar búsquedas de revisiones sistemáticas (Del Olmo, Alcázar y López de Val, 2005). Muy similar es la base de datos de la red Epistemonikos en Chile.

Desde las perspectivas críticas con la MBE, se encuentran los grupos de trabajo que han surgido desde la IT y las $\mathrm{Cl}$. El primero surgió a principios del siglo actual y tuvo como principal interés el diseño de un método bajo el cual la MBE tuviera la posibilidad de ser convalidada por los practicantes y los sujetos de estudio para lograr mayor efectividad en la intervención (Hernández-Carrillo, Campillo y Sánchez-Mendiola, 2018). En México, esta línea ha tenido un particular desarrollo en la formación de profesionistas en nutrición, gracias a la creación del Doctorado en Ciencias de la Nutrición Traslacional en el 2017, alojado en el Centro Universitario de Ciencias de la Salud en la Universidad de Guadalajara.

Por otra parte las $\mathrm{Cl}$, buscando un objetivo similar al de la IT, se han centrado en situar la PBE en el ámbito donde suceden las prácticas, considerando su relación con el sistema de salud local y el contexto socioeconómico como determinantes intermedios y estructurales de la salud (Suárez-Obando, Gómez-Restrepo y Castro-Díaz, 2018).

\section{Producción de información científica actual en Latinoamérica}

A continuación se presentan los resultados de una búsqueda simple en las principales bases de datos de publicaciones: PubMed Central, para la producción científica internacional y REDALyC para indagar información científica en Latinoamérica. Así mismo se indagó información sobre publicaciones y revisiones sistematizadas por la Red Cochrane. El objetivo particular de esta búsqueda fue dar cuenta del proceso de producción de información relacionado con la MBE, NuBE, PBE e IT.

Para todos los buscadores se aplicaron filtros temporales (producción de los últimos cinco años a la fecha) y aquellos que cada plataforma permitiera introducir para lograr mayor especificidad. Las palabras clave para la búsqueda fueron evidence based practice, evidence based medicine, evidence based nutrition y translational research. Se privilegió la búsqueda en inglés para lograr mayor cantidad de resultados, puesto que la

\footnotetext{
* Como Teorías del Consenso, se concentran todas aquellas disciplinas derivadas del enfoque funcionalista, en particular aquellas en donde se abordan los temas de sistemas de salud.
} 
mayoría de los resúmenes incluyen una traducción desde el idioma original. Los resultados se presentan en la Tabla 1.

La tabla demuestra la mayor concentración de las publicaciones en desarrollar información en PBE y la creciente producción de trabajos relacionados con la IT; así mismo hay muy poca producción desde el 2015 relacionada con NuBE y la MBE se ha mantenido en el interés de la comunidad científica. Llama particularmente la atención el hecho de que REDALyC no registre producción en NuBE desde hace seis años, así como la importante concentración de trabajos en Colombia, tanto desde la perspectiva de la MBE como en IT.

\section{Cuadro 1.}

Concentrado de resultados de búsqueda para palabras clave y en plataformas seleccionadas, octubre de 2019.

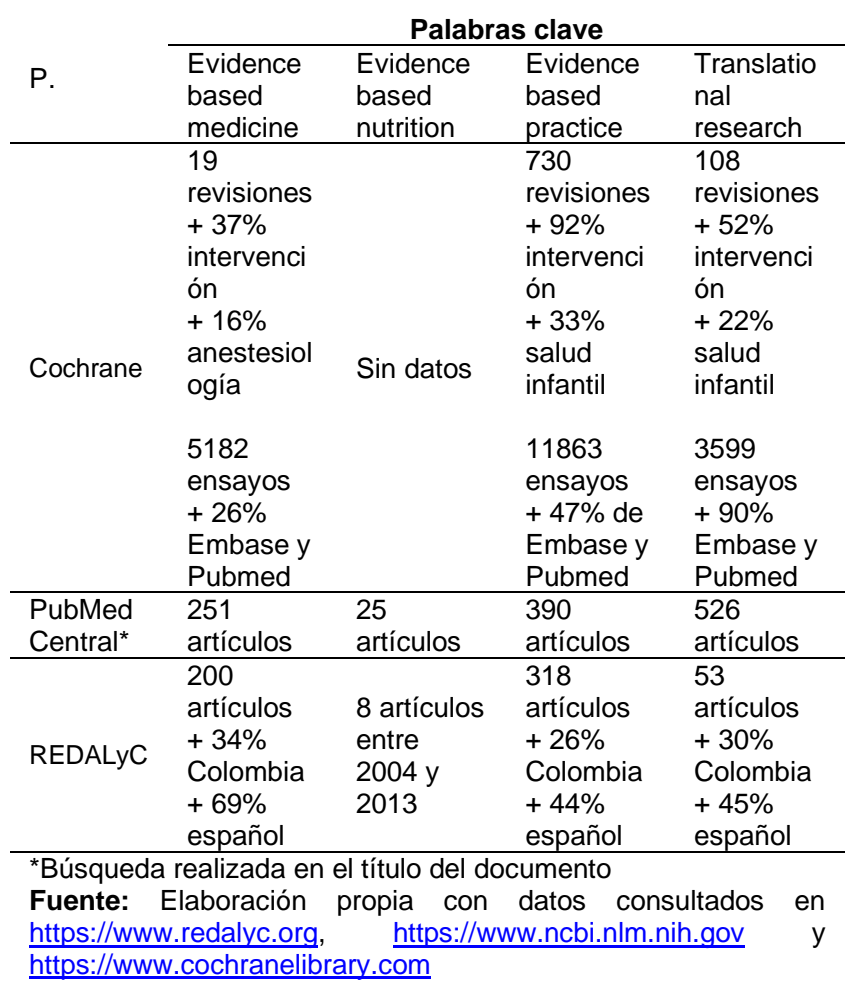

\section{De la evidencia a la práctica; de la práctica a la intervención intercultural}

El elemento sobre el cual se articulan MBE, NuBE y PBE es la evidencia. Las evidencias corresponden a la principal pretensión de la ciencia positiva, específicamente para este caso, desde la epidemiología clínica. La disciplina lleva ya un largo recorrido histórico, pero específicamente se ha desarrollado en el marco del avance de la medicina desde finales del siglo XIX (Alexander, Andre y Fortes, 2018).
Para ser considerada una evidencia, debe de cumplir con una serie de prerrequisitos: mesurabilidad, objetividad, consistencia, especificidad y temporalidad (Cobos-Aguilar, 2016). Todos los anteriores, suponen en conjunto que existe una relación entre la evidencia y su causa, por lo que independientemente del modelo desde el cual se le comprenda (unicausal, multicausal, e incluso, ecológico), estarán estrechamente relacionadas con el paradigma cuantitativo de la ciencia (Crocker-Sagastume, Hunot-Alexander, Moreno-Gaspar, López-Torres, y González-Gutiérrez, 2012).

Sin ánimos de hacer una referencia extensa sobre positivismo, cabe señalar que es ante todo, un modelo de aproximación a la realidad, por lo que asumir a la evidencia como única fuente de saber clínico, supone hacer una reducción. No obstante, la epidemiología ha avanzado cada vez más en el desarrollo de sus modelos, mediante los cuales busca abarcar cada vez más ámbitos de la realidad de la cual emanan (López, Garrido-Latorre y Hernández-Ávila, 2000). Los modelos ecológicos e integral, son un claro ejemplo de cómo se ha buscado reducir el espacio de las causalidades simples para integrar elementos de mayor grado de complejidad en relación con la producción de evidencias socialmente determinadas.

Pese a que la constitución de matrices de determinación de las evidencias puedan ser cada vez más complejas, no eluden a la búsqueda de la verdad científica basada en los datos cuantificables (Alexander, Andre y Fortes, 2018). En materia ética, la evidencia es el componente del principio aristotélico de la verdad, mismo que ha sido ampliamente criticado como eje de la resolución de conflictos ya que, desde el paradigma positivista, se asume como neutro y objetivo. Para la medicina hegemónica, el discurso de la neutralidad y objetividad de la ciencia, derivado de este paradigma, ha sido por demás recurrente y fuertemente relacionado con el avance de la biopolítica (Masana, 2012) y de la descalificación concomitante de otras disciplinas.

El principal movimiento que ha tenido la MBE para tratar de evitar sesgos en la búsqueda por la verdad ha sido pasar de la evidencia científica al metaanálisis y las revisiones sistemáticas, bajo los cuales se pueden organizar distintos niveles de evidencia basados en la fortaleza del método epidemiológico y la plausibilidad estadística (Gómez-Ortega y Amaya-Rey, 2013; Maestre, Ocampo, Useche y Trout, 2012). Sin embargo, esta estrategia no ha sido capaz de superar los retos relacionados con las fuentes de financiamiento de la investigación, los emergentes dilemas éticos y las complicaciones propias en la realización de ensayos clínicos aleatorizados (Del Olmo, Alcázar y López de Val, 2005). 
Así mismo, el afán por centrar la práctica clínica en las evidencias supone que: a) los procesos clínicos basados en las buenas prácticas derivan de forma lineal en acciones en salud y b) las recomendaciones de intervención y diagnóstico clínico son extrapolables a otros campos de atención a la salud.

Habrá que aclarar: generar evidencias y generar acciones en salud son procesos completamente distintos. Las primeras devienen de una forma de generar conocimiento y las segundas pueden surgir de un acto político o como resultante de las relaciones sociales de poder (Alexander, Andre y Fortes, 2018). También, las condiciones de operación del espacio clínico y comunitario son por demás heterogéneas, puesto que están determinadas social y culturalmente (Del Olmo, Alcázar y López de Val, 2005).

Las evidencias son condiciones necesarias, pero no suficientes para responder a las necesidades de la colectividad. Deben por tanto ser concebidas como un elemento adicional dentro de un debate que oriente la toma de decisiones, integrándose a las dimensiones social, cultural y político-económica (Alexander, Andre y Fortes, 2018).

Ante estas demandas del espacio comunitario, la IT y las $\mathrm{Cl}$ han buscado dar cuenta de los procesos de implementación de las PBE, no obstante parten en su mayoría de comprender a los sujetos de forma pasiva. Aunque ambas incluyen como parte del análisis la apropiación de la intervención por parte de la población diana, se excluye la forma mediante la cual las personas construyeron estrategias propias a partir de la información (Suárez, Gómez y Castro, 2018). La información, aunque relaciona fuentes cuantitativas y cualitativas, no está jerarquizada y se utiliza como dato. Así mismo, se supone al contexto como un determinante preexistente e inmoldeable y se pretende que el contexto decante directamente en ciertos retos para la implementación, pero no así se revisan las intermediaciones entre los sujetos y el contexto (Suárez, Gómez y Castro, 2018).

Incluso desde el paradigma de la PBE y la NuBE, se ha demostrado que es incompatible la extrapolación de los resultados clínicos para la población. En una revisión de la Red Cochrane, que buscó la plausibilidad de suplementar nutricionalmente a personas con desnutrición provenientes de grupos vulnerables, encontró importante desestimar los efectos potencialmente benéficos de la alimentación complementaria, puesto que eran para el caso, modestos o escasos. Una de las conclusiones fue resaltar que la desnutrición está íntimamente relacionada con la falta de acceso a los alimentos, por lo que la resolución del problema supera la esfera de la práctica comunitaria. Ante esto, los autores propusieron ampliar la perspectiva de los estudios, sobre todo, de las intervenciones para lograr un espectro temporal más amplio en la satisfacción de las necesidades nutricionales de las poblaciones de estudio (Vissier, McLachlan, Maayan y Garner, 2018).

En otro trabajo del mismo grupo, se indagó sobre los programas de provisión escolar de alimentos, donde encontraron resultados contrastantes entre países de ingresos altos y bajos: en los primeros, la salud física y psicosocial se potencializa, en los segundos los efectos son más discretos (Kristjansson et al., 2007). Estos resultados conducen a la necesidad de incorporar perspectivas críticas sobre la asimetría socioeconómica entre los países, para lo cual son fundamentales los trabajos que desde la Medicina Social (López y Blanco, 1994) y otros autores han denominado como el posicionamiento crítico de las DSS, desde se pueden conocer las relaciones de poder y las dinámicas en la acumulación del capital y la influencia que tienen sobre el proceso de salud y enfermedad que conlleva a patrones de trabajo y de consumo, formas de cultura que conducen a formas malsanas de vivir y llevan a los individuos a padecer la enfermedad de forma diferencial, según clase social, género o etnia. (Verdugo y Reyes, 2017:6).

Siguiendo con las posturas acríticas en PBE, NuBE y MBE, preocupa el énfasis que se ha puesto en la conducta individual, tanto para enarbolarla como principal método para formar prácticas, como para promover esquemas de intervención basados en conductas estándar. Una de las revisiones de Cochrane cuyo objetivo fue demostrar que la toma de decisiones debe basarse más en la efectividad de las intervenciones, concluye que en países de ingresos bajos, el personal de salud tiene mejor efectividad en sus intervenciones al basar su práctica en evidencias. Sin embargo, en el resumen de estrategias se demuestra que la principal estrategia de implementación de la PBE es desarrollar modelos de cambio conductual entre los participantes (Pantoja et al., 2017).

Resalta de esta revisión el peso que tiene la conducta, como un fenómeno individual modificable, dentro del espacio clínico y cuyos cambios pueden desarrollarse de igual forma en países con contextos socioeconómicos y culturales distintos. Sin embargo, las conductas no pueden ser transpuestas de un campo individual a la resolución de problemas del universo comunitario, puesto que la complejidad de los problemas en promoción, protección, recuperación y rehabilitación de la salud, superan el espacio clínico individual (Alexander, Andre y Fortes, 2018), se deben comprender los fenómenos de salud, enfermedad y atención, deben como procesos dialécticos, en donde las particularidades sean observadas a las luz del contexto social que las determina (Verdugo y Reyes, 2017).

Cabe resaltar que los procesos de atención no son el resultado causal de una pauta conductual, sino que 
en conjunto responden a elementos insertos tanto en la infraestructura como en la superestructura (siguiendo a Marvin Harris). En ese sentido, la apropiación de pautas de conducta dependerá también de la hegemonía del modelo médico, que es diferente entre cada sociedad y grupo social. Tratar de instaurar un modelo conductual significa homogenizar los procedimientos y prescindir de componentes émicos que a su vez, engloban los modos en que históricamente, cada sociedad no solo ha generado modelos de atención, sino también, generado conocimiento (Masana, 2012).

La búsqueda por las evidencias, por tanto, debe superar la verticalidad del modelo médico hegemónico (Menéndez, 2003) y construirse desde la interculturalidad, sobre todo en una región tan diversa como América Latina y en particular, México. La intreculturalidad ocurre cuando personas o grupos requieren convivir e interactuar y sus culturas son diferentes... se define como la interacción y el diálogo respetuoso y equilibrado entre personas $o$ grupos con diferentes culturas. Una relación intercultural apropiada o propositiva es aquella que se efectúa de manera voluntaria y con vocación humanista después de una preparación, sensibilización y capacitación que conduce a mejores resultados mediante el diálogo respetuoso, tolerante $y$ flexible entre las personas 0 grupos de las dos culturas. (Vargas, 2006: 179)

Sin embargo, requerir que la NuBE adopte una perspectiva crítica de los problemas de salud y se efectúe desde la intreculutralidad, no supone que se deba de prescindir de los mecanismos que ya ha determinado para establecer un rigor en la selección de la información Por el contrario, se deben de mantener pero con la particularidad de comprender los conocimientos tanto éticos como émicos en igualdad jerárquica y adecuada a la perspectiva personal y social de los que serán beneficiados en lograr mejores prácticas en salud y nutrición, más aún en un espacio comunitario.

\section{Líneas finales}

La formación de los nutriólogos ha estado influenciada por la hegemonía de la medicina, por lo que el ejercicio profesional deberá superar los enfoques clínicos y buscar el desarrollo particular del área desde una comprensión transdcisiplinar de la alimentación. Aunque los métodos de las IT y las $\mathrm{Cl}$ aún no han dado cuenta de las posibilidades de lograr un cambio de paradigma, se debe trabajar integrando las nociones de la NuBE y las PBE al ejercicio de la nutrición desde una perspectiva integral, desde la que se conciban las particularidades interculturales de la comunidad, así como su devenir histórico y los procesos socioeconómicos que las determinan.
El proceso de generación del conocimiento, debe de prescindir del discurso experto de la biomedicina (Masana, 2012) para construirse desde una perspectiva crítica, en donde se asuma que los fenómenos de salud y enfermedad, tanto personales como colectivos, devienen de un modelo económico particular e históricamente determinado. En ese sentido, podrá abonarse el camino para repensar a la evidencia como una construcción cultural que tiene especificidad histórica, social y cultural.

\section{Referencias}

Alcántara Moreno, G. (2008). La definición de salud de la Organización Mundial de la Salud y la interdisciplinariedad. Sapiens. Revista Universitaria de Investigación, 9(1), 93-107.

Alexander, P., Andre, S., Fortes, P.J. (2018). Da prática baseada em evidências para a saúde coletiva informada por evidências: revisão narrativa. Revista Brasileira em Promoção da Saúde, 31(2), 1-7.

Cobos Aguilar, Héctor (2016). Lectura crítica de investigación en educación médica. Investigación en Educación Médica, 5(18), 115120

Crocker-Sagastume, R., Hunot-Alexander, C., Moreno-Gaspar, L.E., López-Torres, P. y González-Gutiérrez, M. (2012). Epistemologías y paradigmas de los campos disciplinares de la nutrición y los alimentos en la formación de nutriólogos. Análisis y propuestas para el desarrollo curricular. Revista de Educación y Desarrollo, 21, 49-58.

Del Olmo, D., Alcázar V. y López de Val, T. (2005). Nutrición basada en la evidencia: presente, limitaciones y futuro. Endocrinología, Diabetes y Nutrición, 52(S2): 2-7.

Foucault, M. (1974). La crisis de la medicina o la crisis de la antimedicina. Educación, medicina y salud, 10(2), 152-170.

Freyermuth, G. y Sesia, P. (2006). Del curanderismo a la influenza aviaria: viejas y nuevas perspectivas de la antropología médica. Desacatos, 20, $9-28$

Garine, I. de. (1999). Antropología de la alimentación: entre Naturaleza y Cultura. Alimentación y Cultura. Actas del Congreso Internacional, (I), pp. 13-34.

Gómez-Ortega, O.R. y Amaya-Rey, M.C.P. (2013). ICrESAI-IMeCI: instrumentos para elegir y evaluar artículos científicos para la investigación y la práctica basada en evidencia. Aquichan, 13(3), 407420 .

Hernández-Carrillo, F., Campillo, M. y Sánchez-Mendiola, M. (2018) Investigación traslacional en ciencias de la salud: implicaciones educativas y retos. Investigación en Educación Médica, 7(28), 85-97.

Kristjansson B., Petticrew M., MacDonald B., Krasevec J., Janzen L., Greenhalgh T., Welch V. (2007). School feeding for improving the physical and psychosocial health of disadvantaged students. Cochrane Database of Systematic Reviews, Issue 1. Art. No.: CD004676. DOI: 10.1002/14651858.CD004676.pub2.

Liscano Pinzón, Y. D. L., y Montoya, J. H. E. (2015). Exploración y análisis a los determinantes sociales estructurales e intermedios de la pandemia del VIH-SIDA. Acta Odontológica Colombiana, 5(1), 117 131.

López Arellano, O; Blanco Gil, J. (1994). Modelos sociomédicos en salud pública: coincidencias y desencuentros. Salud Pública de México, 36(4), 374-384 
López S., Garrido-Latorre F., Hernández-Ávila, M. (2000). Desarrollo histórico de la epidemiología: su formación como disciplina científica. Salud Pública de México, 42 (2): 133-143.

Maestre, J.M., Ocampo, C., Useche, N.S. y Trout, G.O. (2012). Medicina basada en la evidencia: revisión del concepto. Duazary, 9(2), 159-166.

Maldonado-Simó, M.L. (2012). La obesidad comop problema social. Los límites entre lo que es saludable y lo que es estético. En M. Garcia Arnaiz (ed.) Alimentación, salud y cultura: encuentros interdisciplinares (312-338). Tarragona, España: Publicaciones URV.

Masana, L. (2012). Dogma nutricional bajo paradigma biomédico: repercusiones en personas con enfermedades crónicas. En M. Garcia Arnaiz (ed.) Alimentación, salud y cultura: encuentros interdisciplinares (79-100). Tarragona, España: Publicaciones URV.

Menéndez, E.L. (2003). Modelos de atención de los padecimientos: de exclusiones teóricas y articulaciones prácticas, Ciéncia \& Saúde Coletiva, 8(1), 185-207.

Pantoja, T., Opiyo N., Lewin, S., Paulsen, E., Ciapponi, A., Wiysonge, C.S.,...Oxman, A.D. (2017). Implementation strategies for health systems in low-income countries: an overview of systematic reviews. Cochrane Database of Systematic Reviews, Issue 9. Art. No.: CD011086. DOI: 10.1002/14651858.CD011086.pub2.

Sackett, D., Rosenberg, W., Muir Gray, J., Haynes, R. Richardson, W. (1996). Evidence based medicine: what it is and what it isn't. British Medical Journal, 312, 71-72.

Sacristán, J.A. (2013). Evidence based medicine and patient centered medicine: Some thoughts on their integration. Revista Clínica Española, 213, 460-464

Suárez-Obando, F., Gómez-Restrepo, C., Castro-Díaz, S.M. (2018) Ciencias de la implementación: de la evidencia a la práctica. Acta Médica Colombiana, 43(4), 207-216.

Vargas, L.A. (2006). La aplicación de la antropología de la alimentación nspepen contextos interculturales. En Betrán, M. y Arroyo, P. Antropología y nutrición. México: Fundación Mexicana para la Salud: 177-194.

Verdugo, L.M. y Reyes, S.I. (2017). Modelo crítico en Salud para el abordaje de la situación de inseguridad alimentaria: hacia una nueva política alternativa. Margen: revista de trabajo social y ciencias sociales, 85- junio 2017. Disponible en http://www.margen.org/suscri/numero85.html.

Visser, J., McLachlan, M.H., Maayan N. y Garner, P. (2018). Communitybased supplementary feeding for food insecure, vulnerable and malnourished populations - an overview of systematic reviews. Cochrane Database of Systematic Reviews, Issue 11. Art. No.: CD010578. DOI: 10.1002/14651858.CD010578.pub2. 